\title{
Effect of Ginkgo biloba extract on the expressions of Cox-2 and GST-Pi in rats with hepatocellular carcinoma risk
}

\author{
Chao $\mathrm{Ou}^{1}{ }^{*}$, Hai-Ping Zheng ${ }^{2}$, Jian-Jia Su ${ }^{1 *}$, Ji Cao ${ }^{1}$, Guo-Jian $\mathrm{Li}^{2}$, Le-Qun $\mathrm{Li}^{3}$
}

1. Department of Experimental Pathology, The Affiliated Tumor Hospital of Guangxi Medical University, Nanning, Guangxi Province 530021, China

2. Department of Medical Oncology, The First Affiliated Hospital of Guangxi Medical University, Nanning, Guangxi Province 530021, China

3. Department of Hepatobiliary Surgery, The Affiliated Tumor Hospital of Guangxi Medical University, Nanning Guangxi Province 530021, China

*These authors contributed equally.

\begin{abstract}
Background: Hepatocellular carcinoma (HCC) is one of the most common and aggressive cancers worldwide, and the pathogenesis is complicated at present. There iare few effective therapeutic measures, and novel therapeutic strategies are urgently required to improve clinical outcome. Ginkgo biloba extract (EGb) is reported to have an anti-cancer activity.

Objectives: To explore the effect of EGb on expressions of cyclooxygenase-2 (Cox-2) and glutathione S-transferase Pi (GST-Pi) in the pathogenesis of HCC.

Methods: 120 Wistar rats were divided into three groups at random: normal control group (control group), HCC risk group without treatment (HCC risk group), HCC risk group treated with EGb (EGb group); n=40, respectively. The HCC risk in rat was induced by aflatoxin B1 injection. At the end of 13-week, 33-week, 53-week and 73-week, 10 rats in each group were killed and the relevant samples were collected.

Results: The mRNA and protein expressions of Cox-2 and GST-Pi were measured by real-time reverse transcription polymerase chain reaction, immunohistochemical analysis and western-blot. When compared with those in the control group in 73-week, the mRNA and protein expressions of GST-Pi in EGb group were weaker than those in HCC risk group in 73-week. However, the mRNA and protein expressions of Cox-2 in HCC risk group were increased than that of control group, and there was no statistical difference for mRNA and protein expressions of Cox-2 between HCC risk group and EGb group.
\end{abstract}

Conclusion: EGb can regulate the expression of GST-Pi, but it does not seem to have an effect on Cox-2 expression in the liver of HCC risk rats.

Keywords: Hepatocellular carcinoma (HCC); Ginkgo biloba extract (EGb); Cox-2; GST-Pi

African Health Sciences 2014;14(1): 37-48 http://dx.doi.org/10.4314/ahs.v14i1.7

\section{Introduction}

Hepatocellular carcinoma (HCC) is one of the most lethal and prevalent liver $[1,2]$. HCC is highly prevalent in many Asian countries, accounting for $80 \%$ of victims worldwide [3, 4]. Most HCC patients show chronic

$$
\begin{aligned}
& \text { Corresponding Author } \\
& \text { Le-Qun Li: } \\
& \text { Department of Hepatobiliary } \\
& \text { Surgery, The Affiliated Tumor } \\
& \text { Hospital of Guangxi Medical University } \\
& \text { Nanning Guangxi } \\
& \text { Province 530021, China } \\
& \text { E-mail: lequnli@163.com }
\end{aligned}
$$

hepatitis or cirrhosis caused by persistent infection with hepatitis B virus (HBV) or hepatitis $\mathrm{C}$ virus (HCV) [5-7]. Aflatoxin B1 (AFB1) is also very important in the onset of HCC. Hepatectomy is regarded as the optimal curative treatment for HCC at present; however, the postoperative outcome remains unsatisfactory [8]. Its underlying biological mechanism remains unclear and no well-documented drug and targeting has been approved to date [9].

Cyclooxygenase 2 (Cox-2) is expressed highly in cancer and it is found to be implicated in tumor progression, and its inhibition can reduce tumor growth and augment therapy $[10,11]$. Glutathione S-transferase $\mathrm{Pi}$ (GST-Pi) is a subgroup of GST family, which provides cellular protection against free radical and carcinogenic compounds due to its detoxifying function [12]. There 
was no report studying the expressions of Cox- 2 and GST-Pi in the HCC rats induced by AFB1. In this study, we investigated the association of Cox-2 and GST-Pi expressions with HCC risk in rats induced by AFB1.

Ginkgo Biloba is one of the oldest living tree species and it has been described as a living fossil. Its beneficial effects were known 5000 years ago in traditional Chinese medicine. The study of the biological activities of Ginkgo Biloba extract 761 (EGb 761), a standardized extract of Ginkgo Biloba with a well-defined mixture developed more than 20 years ago, has gained great popularity in European countries and the USA [13]. EGb 761 has been standardized to ensure the consistency of its composition and a reliable, safe, efficacy profile [13]. The EGb 761 extract exhibits important properties, such as anti-oxidation, anti-apoptosis and anti-inflammation. At present, some studies [14-16] reports that EGb can take an anti-cancer role. Whether there was an association between EGb and Cox-2 / GST-Pi expression in the HCC risk rats induced by AFB1, there was no any report. In our study, we investigated the alteration of mRNA and protein expressions of Cox-2 and GST-Pi in liver tissue in a rat with HCC risk, treated with EGb to explore the effect of EGb on expressions of Cox-2 and GST-Pi in the pathogenesis of HCC risk.

\section{Materials and methods \\ Animal model}

120 healthy male rats, $180 \mathrm{~g}$ to $200 \mathrm{~g}$, of Wistar backgrounds were purchased from the Experimental Animal center of Guangxi Medical University, Nanning, China. All procedures were approved by the animal ethics committee of Guangxi Medical University. The rats were divided into three groups at random: normal control group (control group), HCC risk group without treatment (HCC risk group), HCC risk group treated with EGb (EGb group); $n=40$, respectively. The risk of HCC in rat was induced by intraperitoneal injection using aflatoxin B1 (AFB1) as follow: 4-7 weeks and 9-12 weeks, $200 \mu \mathrm{g} / \mathrm{kg}$, thrice a week; 14-17 weeks, 19-22 weeks, 24-27 weeks, and 29-32 weeks, $100 \mu \mathrm{g} / \mathrm{kg}$, twice a week; $34-73$ weeks, $100 \mu \mathrm{g} / \mathrm{kg}$, once a week. The rats in EGb group were treated with EGb761 from the first week to the end ( $2 \mathrm{~g} / \mathrm{kg}$.d; Sitexin, co.; Guilin, China). At the end of 13-week, 33-week, 53-week and 73-week, 10 rats in each group were killed and the relevant samples were collected for molecular biology determination.

Real time reverse transcription polymerase chain reaction to detect Cox-2 and GST-Pi mRNA expression in liver tissue
Renal tissue was homogenized and total RNA was extracted with TRIzol (Beijing Tiangen, Co., China). Ultraviolet spectrophotometer measuring absorbance, agarose gel electrophoresis confirmed that there had been no degradation of RNA by visualizing the $18 \mathrm{~S}$ and 28S RNA bands under ultraviolet light [17, 18]. Primers of were designed according to primer design principles by Primer Premier 5.0. The primers for Cox-2, GST-Pi and internal control $\beta$-actin were as follows: F 5'- CCTCGTCCAGATGCTATCT'T-3' and R 5'-GAAGGTGCTAGGTT'TCCAG-3' for Cox2;F5'-AGATGTCTGGCTTCAAGGCT-3'andR5'ATTTGCATCGAAGGTCCTCC-3'forGST-Pi;F5'GCC CCTGAGGAGCACCC'TGT-3' andR 5'ACGCTCGGTCAGGATCTTCA-3' for $\beta$-actin. One microgram total RNA from the liver tissue of each rat was reverse transcribed into cDNA with an ExScript RT reagent kit (Fermentas). Cox-2, GST-Pi and $\beta$-actin were amplified with SYBR Premix Ex Taq (Beijing Tiangen, Co., China). Gene expression of $\beta$-actin was also measured in each sample and used as an internal control for loading and reverse transcription efficiency. The analysis for each sample was performed in triplicate. The average threshold cycle (Ct, the cycles of template amplification to the threshold) was worked out as the value of each sample. The data for fold change was analyzed using $2^{-\Delta \Delta \mathrm{Ct}}[19,20]$.

\section{Immunohistochemical analysis of Cox-2 and GST-Pi}

The operation was implemented using the streptavidinperoxidase immunohistochemical method. Renal tissue samples were fixed in 10\% neutral formaldehyde, dehydrated with ethanol, and embedded in paraffin. Serial $4 \mu \mathrm{m}$ sections were collected sequentially on glass slides. The paraffin was removed from the sections with xylene and rehydrated in graded ethanol. In order to retrieve antigenicity from formalin fixation, we incubated the sections for $10 \mathrm{~min}$ in $10 \mathrm{mmol} / 1$ sodium citrate buffer using a microwave oven. Endogenous peroxidase activity was blocked by further pretreatment with 3\% hydrogen peroxide and methanol. Finally, the sections were incubated with antibody against GSTPi (Thermo Fisher Scientific, Co., Runcorn, UK), Cox-2 (Thermo Fisher Scientific, Co., Runcorn, UK) overnight at $4{ }^{\circ} \mathrm{C}$. The sections were washed thoroughly in phosphate-buffered saline (PBS) solution and incubated with rabbit anti-mouse biotinylated second antibody immunoglobulin (Shanghai Changdao, Co., Inc., China) for $30 \mathrm{~min}$. Finally, the sections were stained with diaminobenizidine (Maixin Bio, Co., Inc., China). 
We obtained negative controls by replacing specific antisera with PBS solution. Brownish yellow granular or linear deposits in the cells or matrix were interpreted as positive areas. Semi-quantitative evaluation was performed by computer-assisted image analysis (Leica Co., Germany). The positive staining of Cox-2 or GSTPi was measured at 400-fold original magnification selected from coded sections of each rat at random.

\section{Western-blot analysis}

The total proteins of liver tissue were extracted using lysing buffer (Beyotime Institute of Biotechnology, China), following a centrifugation at $12000 \mathrm{~g}$ for 10 minutes at $4^{\circ} \mathrm{C}$, and protein content in the supernatant was detected using the BCA protein assay. Equal amounts of extract $(30 \mu \mathrm{g})$ were then separated on an $8 \%$ sodium dodecylsulfate (SDS)-poly-acrylamide gel and transferred by electroblotting to PVDF membranes. The membranes were incubated in $5 \%$ skimmed milk for one hour and were washed with $0.1 \%$ TBST at room temperature, and followed by overnight incubation at $4{ }^{\circ} \mathrm{C}$ with primary antibodies: anti-GSTPi (Thermo Fisher Scientific, Co., Runcorn, UK), antiCox-2 (Thermo Fisher Scientific, Co., Runcorn, UK) and anti- $\beta$-actin (1:5000, Epit Mics). Primary antibodies were diluted using $0.1 \%$ TBST. Alkaline phosphatase conjugated secondary antibodies were incubated with blots for one hour at room temperature. After washing, blots were developed by the ECL Western Blotting detection system, and then exposed to X-ray film for visualization of the protein bands, and membranes were semi-quantified using the Quantity One image analysis system (4.3.1, Bio-Rad, USA). The expression level of GST-Pi or Cox-2 was corrected by comparison with $\beta$-actin, and the final data were expressed used the fold chance.

\section{Statistical analysis}

The data are shown as mean \pm standard deviation (SD). To compare the groups in relation to parameters with normal distribution, One-way analysis of variation (ANOVA) with post-hoc Fisher's LSD (least significant difference) was used. For parameters without normal distribution, Kruskal-Wallis with post-hoc MannWhitney (only for the weight parameter) was used (21, 22). A value of $P<0.05$ was accepted as statistically significant. Statistical analysis was performed using the Statistical Package for Social Sciences (SPSS, version 13.0; Chicago, IL, USA).

\section{Results}

\section{The Cox-2 and GST-Pi mRNA expression in liver tissue}

The mRNA expression of Cox-2 in HCC risk group was similar to that of control group in 13-week or 33-week, and the difference was not statistically significant (all $\mathrm{P}>0.05$; Figure 1). Interestingly, the mRNA expression of Cox- 2 in HCC risk group was notably increased than that of control group in 53-week and 73-week, and the difference was marked (all $\mathrm{P}<0.01$; Figure 1). However, there was no statistical difference for mRNA expression of Cox- 2 between HCC risk group and EGb group in all the time points (all $\mathrm{P}>0.05$; Figure 1). 


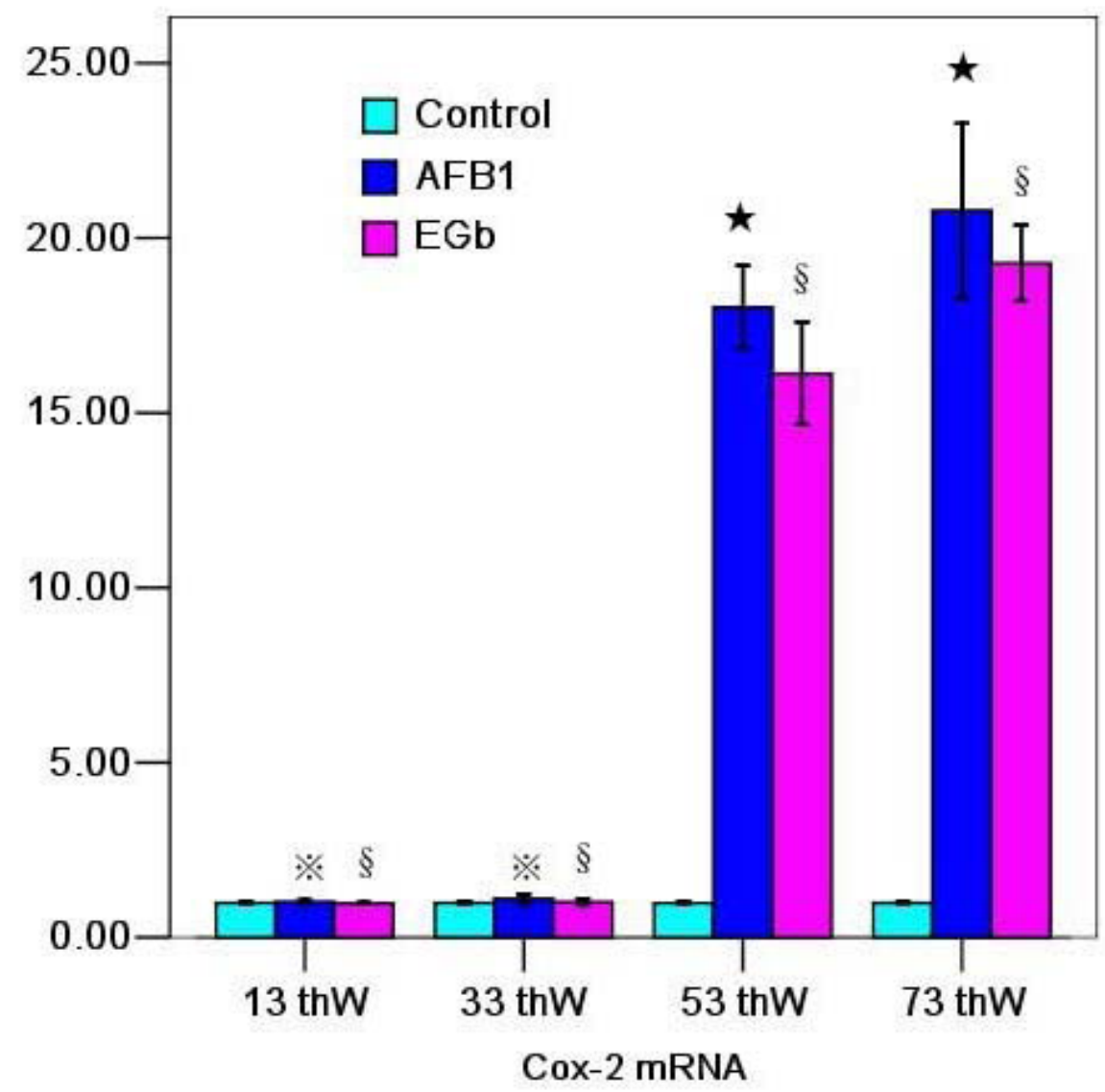

Figure 1 The mRNA expression of Cox-2 in three group. (: $P>0.05$ compared with control group; ): $P>0.01$ compared with control group; §: $P>0.05$ compared with AFB1 group.

When compared with that in control group in all time points, the mRNA expression of GST-Pi in HCC risk group was increased (all $\mathrm{P}<0.01$; Figure 2). Interestingly, the difference of mRNA expression of
GST-Pi between HCC risk group and EGb group was no notably in 13-week, 33-week and 53-week. However, the mRNA expression of GST-Pi in EGb group was marked attenuated than that in HCC risk group in 73week ( $<<0.01$; Figure 2$)$. 


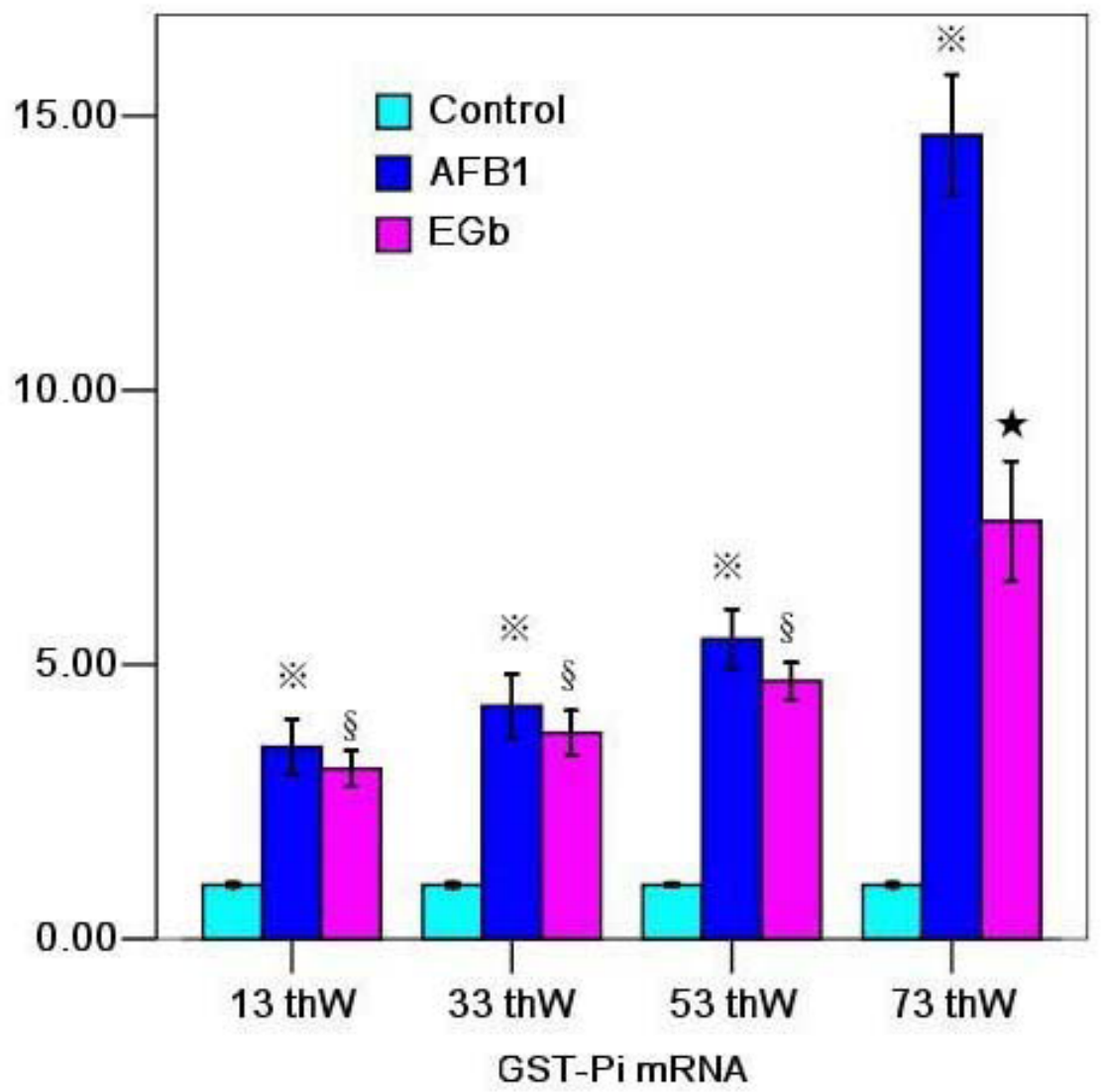

Figure 2 The mRNA expression of GST-Pi in three group. (: $P<0.01$ compared with control group; ): $P>0.01$ compared with AFB1 group; §: $P>0.05$ compared with AFB1 group.

Protein expressions of Cox-2 and GST-Pi in liver tissue using immunohistochemical analysis Immunohistochemicalstaining forCox-2 was performed. The staining for Cox-2 was markedly enhanced in HCC risk group in 53-week or 73-week when compared with those in control group(all $\mathrm{P}<0.01$; Figure 3 ), but the difference was not observed in 13-week or 33-week(all $\mathrm{P}>0.05$; Figure 3). Furthermore, the difference of Cox-2 expression between HCC risk group and EGb group was not observed in 13-week, 33-week, 53-week or 73-week. The typical immunohistochemical staining firgure of for Cox-2 in 73-week was shown in Figure 3. 

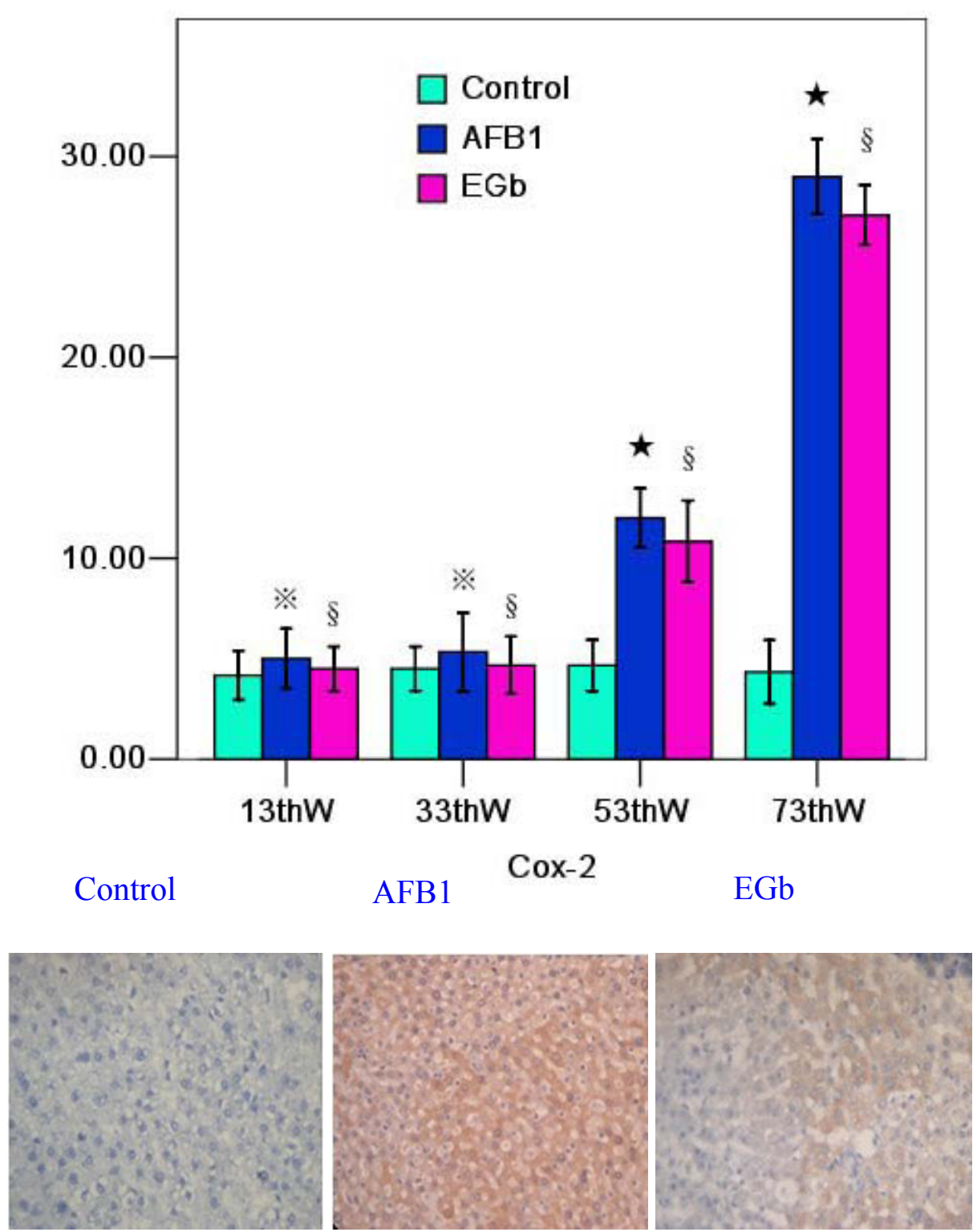

Figure 3 Statistical parameters of the protein expression of Cox-2 and the typical immunohistochemical staining firgure for Cox-2 in three group. The protein expression of Cox- 2 in three group. (: $P>0.05$ compared with control group; ): $P>0.01$ compared with control group; \: $P>0.05$ compared with AFB1 group.

Immunohistochemical staining for GST-Pi was also conducted. The staining for GST-Pi was markedly enhanced in HCC risk group in 13-week, 33-week, 53week or 73-week when compared with those in control group (all $\mathrm{P}<0.01$; Figure 4). The GST-Pi expression in EGb group in 73 -week was marked reduced when compared with that in HCC risk group $(\mathrm{P}<0.01$; Figure
4). However, the difference of GST-Pi expression between HCC risk group and EGb group was not observed in 13-week, 33-week, or 53-week (all P>0.05; Figure 4). The typical immunohistochemical staining firgure of for Cox-2 in 73-week was shown in Figure 4. 

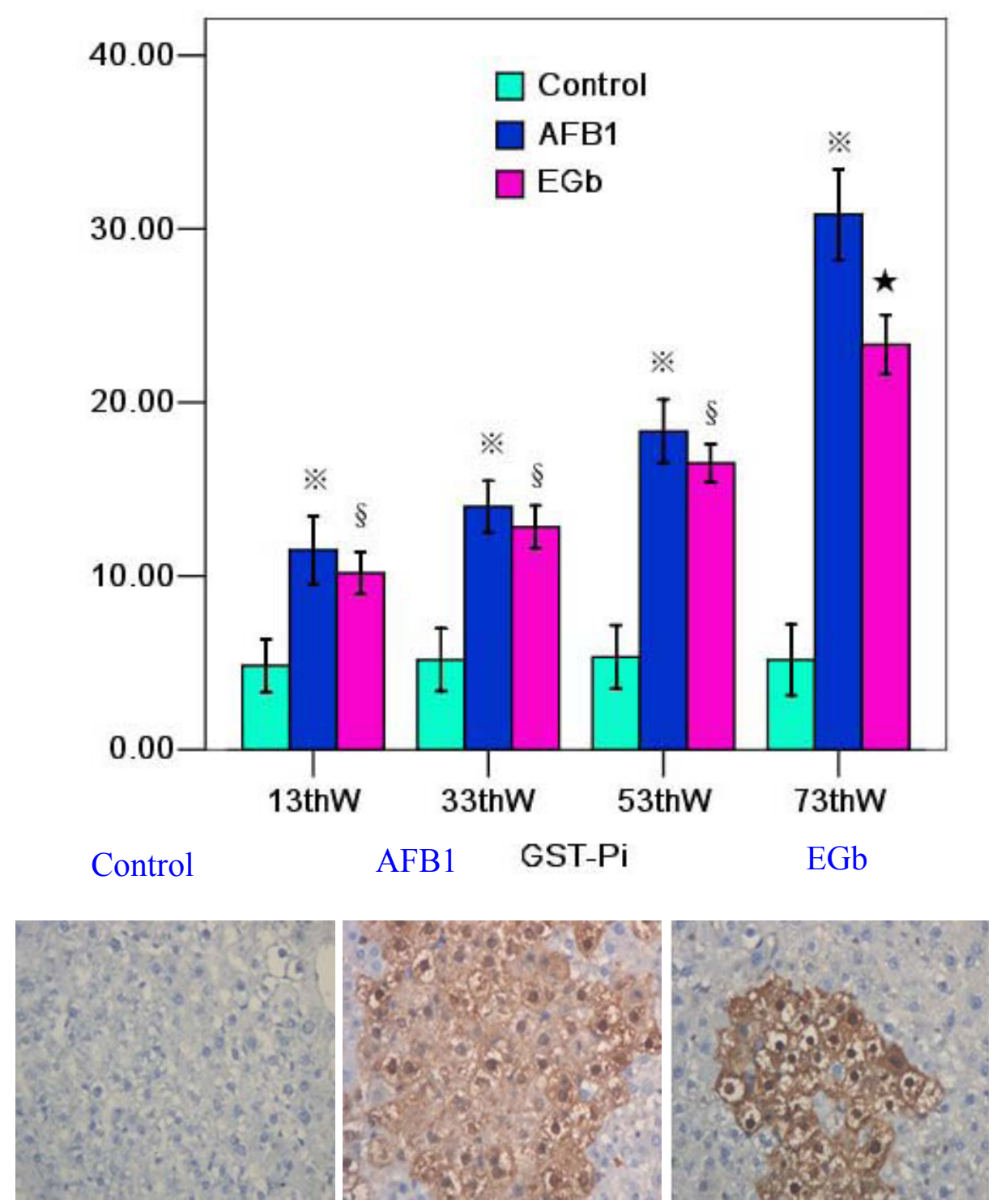

Figure 4 Statistical parameters of the protein expression of GST-Pi and the typical immunohistochemical staining firgure for GST-Pi in three group. (: $P<0.01$ compared with control group; ): $P>0.01$ compared with AFB1 group; S: $P>0.05$ compared with AFB1 group.

Protein expressions of Cox-2 and GST-Pi in liver tissue using western-blot analysis

There was no statistical difference for the protein expression of Cox-2 between HCC risk group and control group in 13-week and 33-week (all $\mathrm{P}>0.05$; Figure 5). Interestingly, the protein expression of Cox-2 in HCC risk group was markedly increased than that of control group in 53-week and 73-week, and the difference was notably (all $\mathrm{P}<0.05$; Figure 5). There was no statistical difference for protein expression of Cox-2 between HCC risk group and EGb group in all the time points (each $\mathrm{P}>0.05$; Figure 5). The typical blot bands for Cox- 2 were presented in Figure 5 

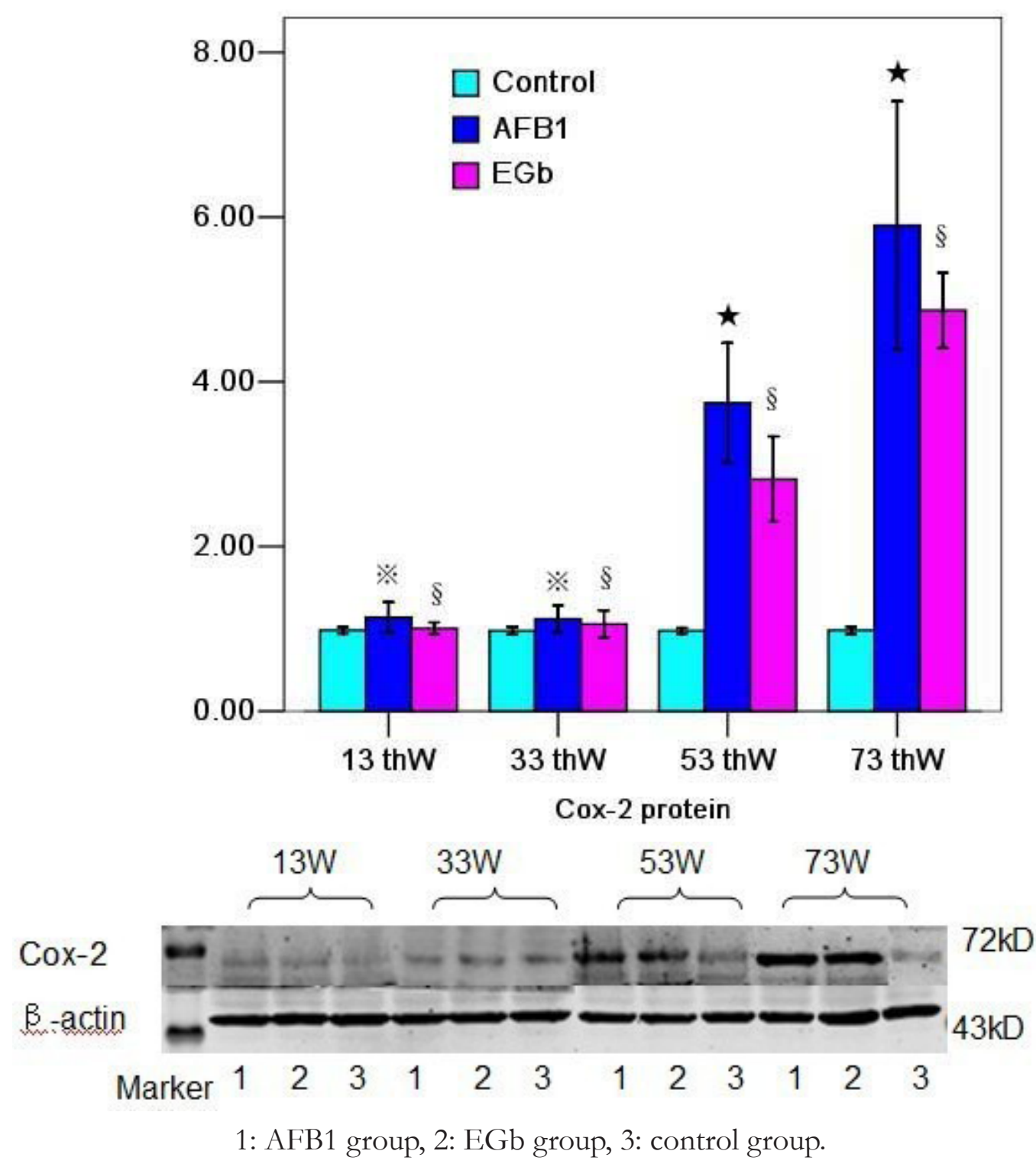

Figure 5 Statistical parameters of the protein expression of Cox- 2 and the typical blot bands for Cox-2 in three group. The protein expression of Cox-2 in three group. (: $P>0.05$ compared with control group; ): $P>0.01$ compared with control group; §: $P>0.05$ compared with AFB1 group.

The protein expression of GST-Pi in HCC risk group was increased than that of control group in all time points (all $\mathrm{P}<0.01$; Figure 6). Interestingly, the difference of protein expression of GST-Pi between HCC risk group and EGb group was no notably in 13-week, 33-week and 53-week. However, the protein expression of GST-Pi in EGb group was marked attenuated than that in HCC risk group in 73-week $(\mathrm{P}<0.01$; Figure 6$)$. The typical blot bands for GST-Pi were presented in Figure 6. 

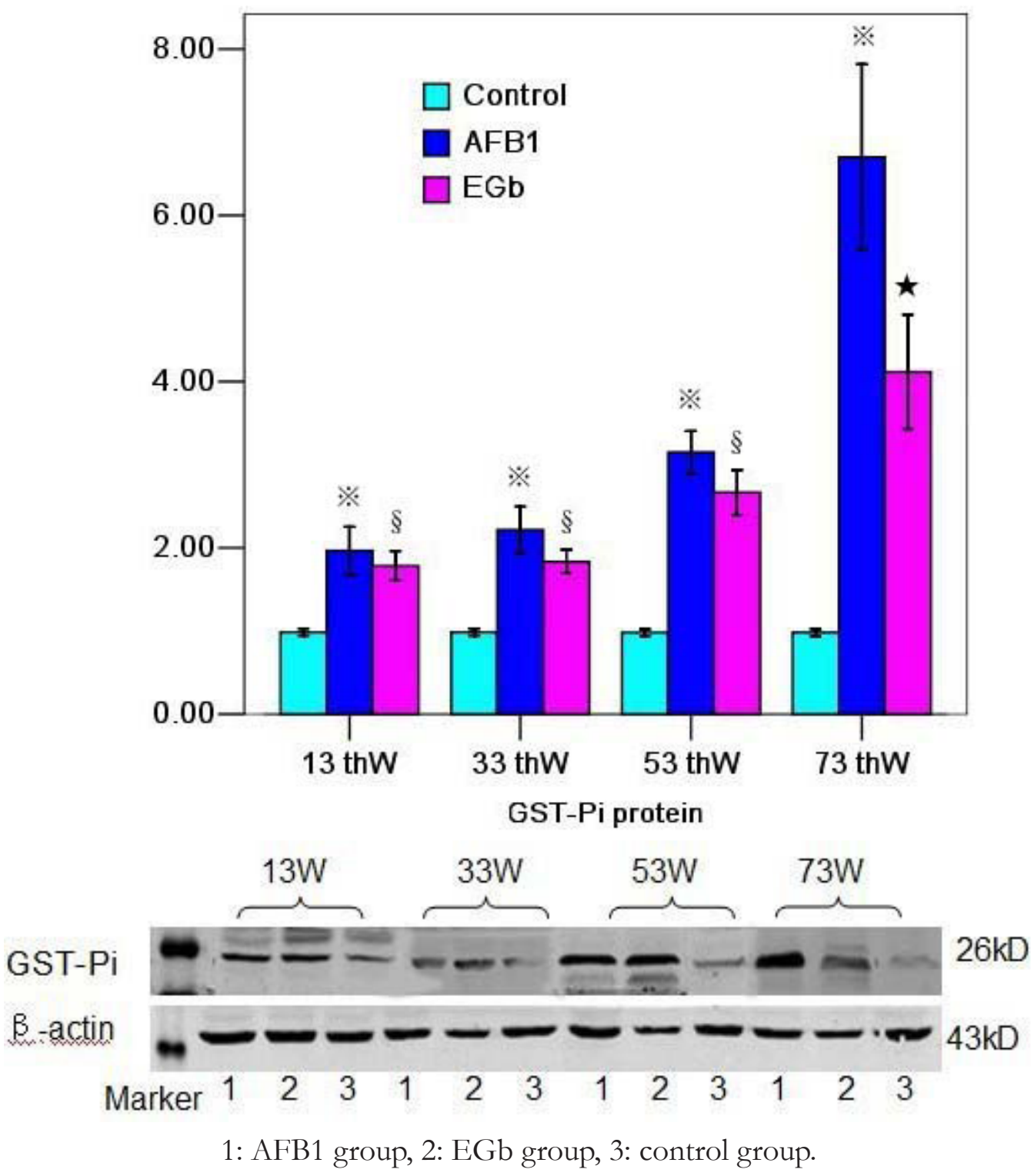

Figure 6 Statistical parameters of the protein expression of GST-Pi and the typical blot bands for GST-Pi in three group. (: $P<0.01$ compared with control group; ): $P>0.01$ compared with AFB1 group; $\int: P>0.05$ compared with AFB1 group.

\section{Discussion}

In this study, we found that Cox-2 expression in the HCC risk group was notably increased when compared with control group. Increased Cox-2 was associated with the risk of HCC in rats. The results from real time reverse transcription polymerase chain reaction, immunohistochemical analysis and westernblot analysis were consistent. Cox-2 might be taking part in the pathogenesis of HCC. Over-expression of Cox-2 has been reported to be associated with hepatocarcinogenesis. Ogunwobi et al. [23] reported that upregulation of Cox-2 could promote epithelialmesenchymal transition and carcinogenesis in vitro, and could take part in the development of HCC. Lee et al. [24] reported that the activation of Cox-2 signaling pathway had particular relevance to HCVassociated HCC in patients with HCV-associated HCC. He et al. [25] reported that over-expression of Cox-2 in noncancerous liver regions was an independent and significant indicator predictive of early recurrence of HCC in patients with HBV-related cirrhosis. Those results mentioned above indicated that over-expression of Cox- 2 was associated with the onset of HCC. It was similar to the conclusion from our study. Cox-2 might take part in the pathogenesis of HCC.

GST-Pi expression was also increased in the HCC risk group than that in control group. In our study, we found that the expression of GST-Pi was increased step by step in the HCC risk group when compared with in control group. The results from real time reverse transcription 
polymerase chain reaction, immunohistochemical analysis and western-blot analysis were consistent. Elevation of GST-Pi was associated with the risk of HCC. There were also some reports finding that there might be a relationship between the expression of GST-Pi and the HCC risk. Yang et al. [26] reported that higher GST-pi expression mediated resistance to cisplatin in HCC cell line. Morsi et al. [27] reported that HCC patients had significantly higher serum GST-Pi. Niu et al. [28] found that the increased GST-Pi might be a marker enzyme for immunohistochemical detection of human HCC and its preneoplastic lesions. The conclusion from those reports mentioned above was consistent with ours. So, increased GST-Pi might be associated with the onset of HCC.

In our study, we found that the EGb had no effect on the expression of Cox-2 in rats. Jang et al. [29] found that Cox-2 expression in the cambial meristematic cells was almost completely suppressed by EGb. Bao et al. [30] reported that EGb could inhibit the production of Cox-2 in myocardial ischemia/reperfusion rats. Park et al. [31] found that EGb could inhibit the LPSinduced Cox-2 in macrophage cell line. Our result was inconsistent with that from the studies mentioned above. However, there was no other report to study the relationship between EGb treatment and Cox-2 expression in progress of HCC. More studies on HCC risk should be performed in the future.

Interestingly, we found that EGb could take a protective role and regulate the expression GST-Pi. The GST-Pi expression was weakened when compared with that in HCC risk group in 73-week. There was only one study reporting the relationship between EGb treatment and GST-Pi expression. Sasaki et al. (32) reported that EGb significantly increased the protein level of GST-Pi in the mouse liver. In our study, we didn't found that EGb could reduce the GST-Pi expression in the rats with HCC risk. Its conclusion was similar to ours. We speculated that the EGb could take the protective role against $\mathrm{HCC}$ risk in rats by reducing the expression of GST-Pi, but not Cox-2.

\section{Conclussion}

The expressions of Cox-2 and GST-Pi were increased in the rats with $\mathrm{HCC}$ risk, and EGb could play a protective role against HCC risk in rats induced by AFB1 by reducing the expression of GST-Pi, but not Cox-2, although the detailed mechanisms were not fully understood. At the moment, EGb is already used in clinical treatment for various diseases, such as thyroid carcinoma, Alzheimer's disease and so on. In this investigation, we have demonstrated that EGb can protect against HCC risk in rats treated with AFB1. This observation might offer some new insights to prevent the onset of HCC. However, cells culture and inhibition of signaling pathway for EGb are needed to be conducted to be sure of its effect and to explore the detailed mechanism.

\section{Acknowledgements:}

This work was supported by grant from the Application Foundation Research Programs of Guangxi Science and Technology (0832009)

\section{Competing Interests:}

The authors have declared no competing interest.

\section{References:}

1. Zhang JL, Qian YB, Zhu LX, Xiong QR. Talin1, a valuable marker for diagnosis and prognostic assessment of human hepatocelluar carcinomas. Asian Pac J Cancer Prev. 2011; 12(12):3265-3269.

2. Dodurga Y, Yonguc GN, Avci CB, Bagci G, Gunduz C, Satiroglu-Tufan NL. Investigation of microRNA expression changes in HepG2 cell line in presence of URG4/URGCP and in absence of URG4/URGCP suppressed by RNA interference. Mol Biol Rep. 2012; 39(12):11119-11124.

3. Han KH, Kudo M, Ye SL, Choi JY, Poon RT, Seong J, Park JW, Ichida T, Chung JW, Chow P, Cheng AL. Asian consensus workshop report: expert consensus guideline for the management of intermediate and advanced hepatocellular carcinoma in Asia. OncologyBasel. 2011; 81(S1):158-164.

4. Chen J, Ma L, Peng NF, Wang SJ, Li LQ. A metaanalysis of the relationship between glutathione $\mathrm{S}$ transferases gene polymorphism and hepatocellular carcinoma in Asian population. Mol Biol Rep. 2012; 39(12):10383-10393.

5. Zhang SG, Song WQ, Gao YT, Yang B, Du Z. CD1d gene is a target for a novel amplicon at 1q22-23.1 in human hepatocellular carcinoma. Mol Biol Rep. 2010; 37(1):381-387.

6. Khaled IA, Mahmoud OM, Saleh AF, Bioumie EE. Prevalence of HBV genotypes among Egyptian hepatitis patients. Mol Biol Rep. 2011; 38(7):4353-4357.

7. Gu X, Wang H, Wang A, Dou T, Qi P, Ji Q, Li H, Gao

C. An intronic polymorphism rs2237062 in the CXCL14 
gene influences HBV-related HCC progression in Chinese population. Mol Biol Rep. 2012(2); 39:797-803.

8. Liu H, Zhang A, Qian N, Gao L, Xu L, Zhang W, Jiang K, Cai S, Huang Z, Dong J. Postoperative Transarterial Chemoembolization Benefits Patients with High gamma-Glutamyl Transferase Levels after Curative Hepatectomy for Hepatocellular Carcinoma: A Survival Stratification Analysis. Tohoku J Exp Med. 2012;227:269-280.

9. Lachenmayer A, Alsinet C, Savic R, Cabellos L, Toffanin S, Hoshida Y, Villanueva A, Minguez B, Newell P, Tsai HW, Barretina J, Thung S, Ward SC, Bruix J, Mazzaferro V, Schwartz M, Friedman SL, Llovet JM. Wntpathway activation in two molecular classes of hepatocellular carcinoma and experimental modulation by sorafenib. Clin Cancer Res. 2012; 18(18):4997-5007.

10. Kirane A, Toombs JE, Ostapoff KT, Carbon JG, Zaknoen S, Braunfeld JB, Schwarz RE, Burrows FJ, Brekken RA. Apricoxib, a novel inhibitor of COX-2 markedly improves standard therapy response in molecularly defined models of pancreatic cancer. Clin Cancer Res. 2012;18(18):5031-5042.

11. Song T, Zhang X, Wang C, Wu Y, Dong J, Gao J, Cai W, Hong B. Expression of miR-143 reduces growth and migration of human bladder carcinoma cells by targeting cyclooxygenase-2. Asian Pac J Cancer Prev. 2011; 12(4):929-933.

12. Wang Z, He W, Yang G, Wang J, Wang Z, Nesland JM, Holm R, Suo Z. Decreased expression of GST pi is correlated with a poor prognosis in human esophageal squamous carcinoma. BMC Cancer. 2010; 10:352.

13. Rojas P, Montes P, Rojas C, Serrano-Garcia N, Rojas-Castaneda JC. Effect of a phytopharmaceutical medicine, Ginko biloba extract 761, in an animal model of Parkinson's disease: Therapeutic perspectives. Nutrition. 2012; 28(11-12):1081-1088.

14. Wang WW, Guo JC, Xun YH, Xiao LN, Shi WZ, Shi JP, Lou GQ. The effect of extract of ginkgo biloba leaf during the formation of HBV-related hepatocellular carcinoma. Zhonghua Shi Yan He Lin Chuang Bing Du Xue Za Zhi. 2011; 25(4):325-327.

15. Chen XH, Miao YX, Wang XJ, Yu Z, Geng MY, Han YT, Wang LX. Effects of Ginkgo biloba extract EGb761 on human colon adenocarcinoma cells. Cell Physiol Biochem. 2011; 27(3-4):227-232.

16. Babich H, Ackerman NJ, Burekhovich F, Zuckerbraun HL, Schuck AG. Gingko biloba leaf extract induces oxidative stress in carcinoma HSC-2 cells. Toxicol In Vitro. 2009; 23(6):992-999.
17. Zhou TB, Qin YH, Lei FY, Su LN, Zhao YJ, Huang WF. apoE expression in glomerulus and correlation with glomerulosclerosis induced by adriamycin in rats. Ren Fail. 2011; 33(3):348-354.

18. Zhou TB, Qin YH, Li ZY, Xu HL, Zhao YJ, Lei FY. All-trans retinoic Acid treatment is associated with prohibitin expression in renal interstitial fibrosis rats. Int J Mol Sci. 2012; 13(3):2769-2782.

19. Zhou TB, Qin YH, Lei FY, Su LN, Zhao YJ, Huang WF. All-trans retinoic acid regulates the expression of apolipoprotein $\mathrm{E}$ in rats with glomerulosclerosis induced by Adriamycin. Exp Mol Pathol. 2011; 90(3):287294.

20. Zhou TB, Qin YH, Lei FY, Su LN, Zhao YJ, Huang WF. Less gelatinases is associated with apolipoprotein $\mathrm{E}$ accumulation in glomerulosclerosis rats. Histol Histopathol. 2012; 27(2):249-256.

21. Piroozmand A, Hassan ZM. Evaluation of natural killer cell activity in pre and post treated breast cancer patients. J Cancer Res Ther. 2010; 6(4):478-481.

22. Zhou TB, Qin YH, Ou C, Lei FY, Su LN, Huang WF, Zhao YJ. All-trans retinoic acid can regulate the expressions of gelatinases and apolipoprotein $\mathrm{E}$ in glomerulosclerosis rats. Vascul Pharmacol. 2011; 55(56):169-177.

23. Ogunwobi OO, Liu C. Hepatocyte growth factor upregulation promotes carcinogenesis and epithelialmesenchymal transition in hepatocellular carcinoma via Akt and COX-2 pathways. Clin Exp Metastasis. 2011; 28(8):721-731.

24. Lee JC, Tseng CK, Wu SF, Chang FR, Chiu CC, Wu YC. San-Huang-Xie-Xin-Tang extract suppresses hepatitis $C$ virus replication and virus-induced cyclooxygenase-2 expression. J Viral Hepat. 2011; 18(7):315-324.

25. He YF, Jin J, Wei W, Chang Y, Hu B, Ji CS, Jia WD, Wang XQ, Chen K, Chen J. Overexpression of cyclooxygenase-2 in noncancerous liver tissue increases the postoperative recurrence of hepatocellular carcino$\mathrm{ma}$ in patients with hepatitis B virus-related cirrhosis. Can J Gastroenterol. 2010; 24(7):435-440.

26. Yang JX, Luo Y, Qiu HM, Tang WX. Characterization and resistance mechanisms of cisplatin-resistant human hepatocellular carcinoma cell line. Saudi Med J. 2009;30(1):35-40.

27. Morsi MI, Hussein AE, Mostafa M, El-Abd E, ElMoneim NA. Evaluation of tumour necrosis factoralpha, soluble P-selectin, gamma-glutamyl transferase, glutathione S-transferase-pi and alpha-fetoprotein in patients with hepatocellular carcinoma before and dur- 
ing chemotherapy. Br J Biomed Sci. 2006; 63(2):74-78. 28. Niu ZS, Wang M. Expression of c-erbB-2 and glutathione S-transferase-pi in hepatocellular carcinoma and its adjacent tissue. World J Gastroenterol. 2005; 11(28):4404-4408.

29. Jang SH, Lee EK, Lim MJ, Hong NJ, Oh IS, Jin YW, Jeong HS, Jeong YS, Lee JC, Jang YS. Suppression of lipopolysaccharide-induced expression of inflammatory indicators in RAW 264.7 macrophage cells by extract prepared from Ginkgo biloba cambial meristematic cells. Pharm Biol. 2012; 50(4):420-428.

30. Bao YM, Liu AH, Zhang ZX. Effect of precondition with GBE50 and Salviae miltionrrhizae on cycloxygenase- 2 and its downstream effectors contents in ischemia/reperfusion myocardium. Zhongguo Zhong Xi Yi Jie He Za Zhi. 2010; 30(5):1056-1060.

31. Park YM, Won JH, Yun KJ, Ryu JH, Han YN, Choi SK, Lee KT. Preventive effect of Ginkgo biloba extract (GBB) on the lipopolysaccharide-induced expressions of inducible nitric oxide synthase and cyclooxygenase- 2 via suppression of nuclear factor-kappaB in RAW 264.7 cells. Biol Pharm Bull. 2006;29(5):985-990.

32. Sasaki K, Hatta S, Wada K, Ueda N, Yoshimura T, Endo T, Sakata M, Tanaka T, Haga M. Effects of extract of Ginkgo biloba leaves and its constituents on carcinogen-metabolizing enzyme activities and glutathione levels in mouse liver. Life Sci. 2002;70(14):16571667. 